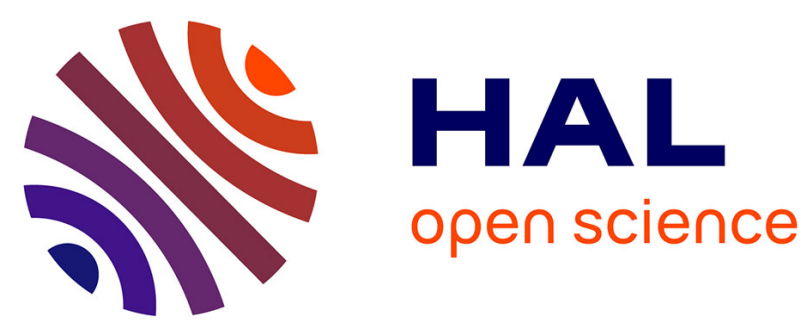

\title{
Values on regular games under Kirchhoff's laws
}

Fabien Lange, Michel Grabisch

\section{To cite this version:}

Fabien Lange, Michel Grabisch. Values on regular games under Kirchhoff's laws. Mathematical Social

Sciences, 2009, 58 (3), pp.322-340. 10.1016/j.mathsocsci.2009.07.003 . halshs-00496553

\section{HAL Id: halshs-00496553 \\ https://shs.hal.science/halshs-00496553}

Submitted on 30 Jun 2010

HAL is a multi-disciplinary open access archive for the deposit and dissemination of scientific research documents, whether they are published or not. The documents may come from teaching and research institutions in France or abroad, or from public or private research centers.
L'archive ouverte pluridisciplinaire HAL, est destinée au dépôt et à la diffusion de documents scientifiques de niveau recherche, publiés ou non, émanant des établissements d'enseignement et de recherche français ou étrangers, des laboratoires publics ou privés. 


\section{Elsevier Editorial System(tm) for Mathematical Social Sciences Manuscript Draft}

Manuscript Number: MSS-D-08-00197R1

Title: Values on regular games under Kirchhoff's laws

Article Type: Research Paper

Keywords: Regular set system; communication situation; regular game; Shapley value; Kirchhoff's laws.

Corresponding Author: Dr Fabien Lange, Ph.D.

Corresponding Author's Institution: Keleti Faculty of Economics

First Author: Fabien Lange, Dr

Order of Authors: Fabien Lange, Dr; Michel Grabisch, $\mathrm{Pr}$

Abstract: The Shapley value is a central notion defining a rational way to share the total worth of a cooperative game among players. We address a general framework leading to applications to games with communication graphs, where the feasible coalitions form a poset whose all maximal chains have the same length. Considering a new way to define the symmetry among players, we propose an axiomatization of the Shapley value of these games. Borrowing ideas from electric networks theory, we show that our symmetry axiom and the efficiency axiom correspond to the two Kirchhoff's laws in the circuit associated to the Hasse diagram of feasible coalitions. 
Fabien LANGE

Keleti Faculty of Economics - Budapest Tech

Tavaszmező u. 15-17

1084 Budapest, Hungary

E-mail: fabien.Lange@kgk.bmf.hu

July 17, 2009

Manuscript MSS-D-08-00197

Dear Professor,

Please find a slight revision of our manuscript entitled "Values on regular games under Kirchhoff's laws", based on the reviewer's comments.

The first author apologizes for the delay of resubmission.

We thank both reviewers for their work and valuable remarks, as well as the Associate Editor.

Yours sincerely,

Fabien Lange 


\title{
Manuscript MSS-D-08-00197: Values on regular games under Kirchhoff's laws
}

\author{
Fabien Lange Michel Grabisch
}

July 17, 2009

We thank both reviewers for their work and valuable remarks, as well as the Associate Editor. Below is the list of changes and argued rebuttals.

\section{Reviewer \#2}

- Another definition of regular set systems is proposed: $\mathcal{N}$ is regular iff there is a set $\mathcal{O}$ of strict orders of $N$ s.t. $\mathcal{N}$ contains precisely all initial segments of orderings in $\mathcal{O}$. The "if part" is wrong: for instance, if $N=\{1, \ldots, 4\}$ and $\mathcal{O}:=\{(1,2,3,4) ;(3,4,1,2)\}$, the resulting $\mathcal{N}$ does not satisfy the definition $(\{3\} \prec\{1,2,3\}$ but $|\{1,2,3\} \backslash\{3\}| \neq 1)$.

- We quite disagree with the idea that the statement of the regularity axiom is too long, and the proposed restatement does not enable to understand its intuition. As specified, this axiom means that for an equidistributed game $v$, the sum of marginal contributions of players should not depend on the considered maximal chain. For such a game, the path taken from coalition $A$ to $B$ has no effect on the successive increasing worth $v(C)$ ( $A \subseteq C \subseteq B$ ), so that our statement of the axiom is defended.

- It is true that the "Shapley-Kirchhoff value" is not explicitely presented. Actually, it appears that the coefficients are computable only for a given regular set sytem, since a linear system has to be solved, where equations depend on the structure of the system. By the way, it is false to say that this value is the arithmetic average of the order values of Weber over all orderings in $\mathcal{O}$. This is actually a compatible-order value, and consequently an average of the order values, but the mean is not arithmetic (coefficients are not the same, depending on the ordering, and may be negative as a corollary of the counterexample of Annex B).

- Theorem 12 does not show that the SK value is monotonic (which is false, according to the aforementionned counterexample), it shows its aggregate monotonicity. 


\section{Reviewer \#1}

- About the fact that we do not give very much support to the claim that the class of regular set systems is natural: in addition of the example of communication situations, we added after Proposition 1 a paragraph with another approach in the process of building regular set systems : by considering permitted/forbidden permutations. The introduction is also amended (p. 2, paragraph 1).

- Technical points p. 8 and p. 12: these are now corrected. 


\title{
Values on regular games under Kirchhoff's laws
}

\author{
Fabien Lange \\ Keleti Faculty of Economics \\ Budapest Tech, Tavaszmező 15-17 \\ 1084 Budapest, Hungary \\ email fabien. lange@kgk. bmf.hu
}

\author{
Michel Grabisch \\ Centre d'Economie de la Sorbonne \\ UMR 8174, CNRS-Université Paris 1 \\ 106-112 bd de l'Hôpital, 75013 Paris, France \\ email michel.grabisch@univ-paris1.fr
}

\begin{abstract}
The Shapley value is a central notion defining a rational way to share the total worth of a cooperative game among players. We address a general framework leading to applications to games with communication graphs, where the feasible coalitions form a poset whose all maximal chains have the same length. Considering a new way to define the symmetry among players, we propose an axiomatization of the Shapley value of these games. Borrowing ideas from electric networks theory, we show that our symmetry axiom and the efficiency axiom correspond to the two Kirchhoff's laws in the circuit associated to the Hasse diagram of feasible coalitions.
\end{abstract}

Key words. Regular set system; communication situation; regular game; Shapley value; Kirchhoff's laws.

\section{JEL code: $\mathrm{C} 71$}

\section{Introduction}

The value or solution concept of a game is a key concept in cooperative game theory, since it defines a rational imputation given to the players if they join the grand coalition. In this respect, the Shapley value remains the best known solution concept (Shapley (1953); Weber (1988)) applied also to more general notions of game, like multichoice games (Grabisch and Lange (2007); Hwang and Liao (2008)).

In the above cited classical works, it is assumed that any coalition of players can form. However, this assumption is often irrealistic, for various reasons (incompatibilities between players, precedence constraints, etc.). A great deal of work has been done in order to consider weaker assumptions on the set of feasible coalitions. Along this line, we may cite Faigle $(1989 ; 1992)$ who introduced the idea of precedence constraints among players, and Bilbao and Edelman, considering that the set of feasible coalitions is a convex geometry. Due to well known results in lattice representation, the construction of Faigle amounts to have a distributive lattice as the set of feasible coalitions, and hence is a particular case of Bilbao and Edelman's construction.

Despite the mathematical interest of convex geometries, we may argue if they fit or not to the framework of game theory. Specifically, feasible coalitions of a convex geometry should satisfy two conditions: (1) if $S$ is a feasible coalition, then it is possible to find a player $i$ such that $S \cup i$ is still feasible, (2) if $S, T$ are feasible, then their intersection too should be feasible. The first condition is a natural and very weak one in a context where the grand coalition can form, since it says that from a given coalition, it is possible to augment it gradually to reach the grand coalition. On the contrary, it is more difficult to accept the second one. 
We propose to consider more general structures, avoiding the closure under intersection, which we call regular set systems, which more or less amounts to take condition (1) above and a symmetric one, saying that from a given coalition $S$, it is possible to withdraw one player while remaining feasible. Regular set systems have been proposed by Honda and Grabisch (2006), and are those set systems having all their maximal chains of length $n$, the number of players. This characterization gives an interesting interpretation in terms of cooperative games, more precisely, of coalition formation: since maximal chains correspond bijectively to permutations (or total orders) on $N$, the set of players, a regular set system restricts the set of allowable or feasible permutations on players, put differently, it restricts the way players can enter the game. One of the main mathematical advantages of regular set systems is that they allow to keep many classical notions defined for games, capacities (Choquet (1953)) and other set functions (Grabisch, Marichal, and Roubens (2000)), such as the Möbius transform, the core, the Shapley value, the entropy, etc., since all these notions can be defined through maximal chains. A general view of regular set systems, giving connections with more classical ordered structures, is given in Section 2.

We first highlight the strong economic interest of defining games on regular set systems on potential economics situations. Indeed, we show that games defined from a connected communication graph (Myerson (1977)) representing the communications links between the players, lead to coalition structures which are regular set systems. Our main aim is the axiomatization of a solution concept for games defined on regular set systems - which we call regular games-, close to the Shapley value. In Section 3, we begin by considering probabilistic and marginalist values, and we generalize results obtained by Bilbao and Edelman. In Section 4, we propose a substitute for the classical symmetry axiom, which cannot be straightforwardly generalized in such general coalition structures. Our proposal, called the regularity axiom, has a more natural interpretation than the hierarchical strength axiom of Faigle and Kern (1992), which is merely a combinatorial axiom. Our main achievement is Theorem 6 , which shows that there is a unique marginalist value satisfying the regularity axiom and efficiency. This is done through an analogy with networks and electrical circuits, explained in Section 5. The efficiency and regularity axioms are shown to be respectively equivalent to the first and second Kirchhoff's laws. A last section is devoted to the study of monotonicity. It is shown that our value does not satisfy the monotonicity axiom in general, but a weaker form of monotonicity, which is the aggregate monotonicity.

In the paper, $N:=\{1,2, \ldots, n\}$ refers to the finite set of players. In order to avoid heavy notations, we will often omit braces for subsets, by writing $i$ instead of $\{i\}$ or 123 for $\{1,2,3\}$. Furthermore, cardinalities of subsets $S, T, \ldots$ will be denoted by the corresponding lower case letters $s, t, \ldots$

\section{Regular games}

Classical cooperative games on $N$ deal with situations where all coalitions of players are feasible. Myerson has been the first who introduced coalition structures where only a subset of the power set of players represent valid coalitions. These latter are defined from a communication situation between the players, leading to the communication graph.

Let $(N, L)$ be a graph, where the vertices represent the players, and the edges denote the possibility to communicate between the players. As a result, the feasible coalitions consist of all subsets of $N$ for which the induced subgraph is connected. In other words, these coalitions are those corresponding to the communication situations between the players: $A \subseteq N$ is feasible if and only if there is a communication chain for any pair of players of $A$. More details about games with communication graphs may be found in Calvo, Lasage, and Nouweland (1999) and 
van den Brink, van der Laan, and Pruzhansky (2004).

For instance, let $n:=3$ and the following communication situation between the players: player 1 is connected to player 2 , and player 2 is connected to player 3 . Then the set of feasible coalitions is $\{\emptyset, 1,2,3,12,23, N\}$.

In all the paper, we are only interested by situations where all players can cooperate together, that is to say, $N$ is a feasible coalition. Hence $(N, L)$ is connected.

Let us consider $\mathcal{N}$ a subcollection of the power set $2^{N}$ of $N$. Then we call $(N, \mathcal{N})$ a set system on $N$ if $\mathcal{N}$ contains $\emptyset$ and $N$. In the sequel, $(N, \mathcal{N})$ always denotes a set system.

Elements of $\mathcal{N}$ are called (feasible) coalitions. For any two coalitions $A, B$ of $\mathcal{N}$, we say that $A$ is covered by $B$, and write $A \prec B$, if $A \subsetneq B$ and $A \subseteq C \subsetneq B$, with $C \in \mathcal{N}$, implies $C=A$.

Definition $1(N, \mathcal{N})$ is a regular set system if it satisfies the following property:

$\forall S, T \in \mathcal{N}$ such that $S \prec T$ in $\mathcal{N}$, then $|T \backslash S|=1$.

If in addition, the regular set system has a lattice structure, then we call it a regular set lattice.

For any two coalitions $S, T$ in a set system $(N, \mathcal{N})$, we call maximal chain from $S$ to $T$ any sequence $\left(S_{0}, S_{1}, \ldots, S_{m}\right)$ of elements of $\mathcal{N}$ such that $S_{0}=S, S_{m}=T$, and $S_{i} \prec S_{i+1}$ for every $0 \leq i \leq m-1$. If $S$ and $T$ are not specified, maximal chains are understood to be from $\emptyset$ to $N$. Note that we find in Honda and Grabisch (2006) Definition 1 under the equivalent form (ii) below:

Proposition 1 Let $(N, \mathcal{N})$ be a set system. Then the following assertions are equivalent:

(i) $(N, \mathcal{N})$ is a regular set system.

(ii) All maximal chains of $(N, \mathcal{N})$ have length $n$, i.e., all maximal chains have exactly $n+1$ elements.

Proof: Assuming that $(N, \mathcal{N})$ is regular, let $C$ be a maximal chain of $(N, \mathcal{N})$. Every element of $C$ covers the previous one, and then contains only one extra player. Thus $C$ contains $n+1$ elements. Conversely, if $(N, \mathcal{N})$ is not regular, i.e. there are two elements $S, T$ such that $S \prec T$ and $|T \backslash S| \geq 2$, then any maximal chain going through $S$ and $T$ has necessarily less than $n+1$ elements.

Clearly, the maximal chains correspond to permutations of $N$, that is, total orders on players. In the classical case, any permutation is possible (that is, the players can enter the game in any order), leading to the regular set system $2^{N}$, the set of all possible coalitions. However, for a regular set system, some permutations may be forbidden. Taking again the case of $N=$ $\{1,2,3\}$, the collection $\{\emptyset, 1,2,13,23,123\}$ is a regular set system, where among the 6 possible permutations, only 1,3,2 and 2,3,1 are permitted. Another interesting interpretation of regular set systems is given below.

EXAMPLE Let $(N, L)$ be a connected communication graph. Then the resulting set system of feasible coalitions is a regular set sytem.

The converse is clearly not true: a regular set system does not necessarily correspond to a communication situation. A necessary (but not sufficient) condition is that all coalitions consisting of one player, are feasible.

Note that regular set systems also satisfy the following properties, which straightforwardly derive from the definition: 
(iii) One-point extension: $\forall S \in \mathcal{N}, S \neq N, \quad \exists i \in N \backslash S$ such that $S \cup i \in \mathcal{N}$.

(iv) Accessibility: $\forall T \in \mathcal{N}, T \neq \emptyset, \exists j \in T$ such that $T \backslash j \in \mathcal{N}$.

These properties are not sufficient to characterize regular set systems, and are actually used by Labreuche as an underlying structure of games in Labreuche (2002).

What is interesting for the sequel, the set of regular set systems is a general class embodying some classical structures such as distributive lattices and convex geometries (Bilbao and Edelman (2000)). We now present them.

A Jordan-Dedekind poset is any poset such that all its maximal chains between any two elements have the same length. Note that if the Jordan-Dedekind poset has least and greatest elements, it is sufficient to verify that all its maximal chains between them have the same length. Thus we call Jordan Dedekind set system any Jordan-Dedekind poset which is a set system. A convex geometry is any set system $(N, \mathcal{N})$ satisfying

(C1) One-point extension property.

(C2) Intersection closure: $\forall A, B \in \mathcal{N}, \quad A \cap B \in \mathcal{N}$.

The dual set system of the convex geometry is called antimatroid, that is to say any set system satisfying

\section{(A1) Accessibility property.}

\section{(A2) Union closure.}

A lattice is distributive when the infimum and the supremum obey the distributivity law. For any poset $(P, \leqslant)$, a subset $Q \subseteq P$ is called a downset of $(P, \leqslant)$ if $x \leqslant y$ and $y \in Q$ imply $x \in Q$. We denote by $\mathcal{O}(P)$ the set of all downsets of $P$. Besides, a join-irreducible element $x$ of a lattice $(L, \leq)$ is an element that is not the least one, and for which $(x=y \vee z)$ implies $(x=y$ or $x=z)$. It is known that the set of all downsets of $(P, \leqslant)$ endowed with the inclusion relation is a distributive lattice. Conversely, a fundamental Theorem due to Birkhoff (1967) says that any distributive lattice $(L, \leq)$ is isomorphic to the set $\mathcal{O}(\mathcal{J})$ of all downsets of the set $\mathcal{J}$ of join-irreducible elements of $L$. Consequently, for any distributive lattice $(L, \leq)$, there is a poset $(P, \leqslant)$ such that $(L, \leq)$ has the isomorphic form $\mathcal{O}(P)$. Moreover, it is also known that distributive lattices which are set systems coincide with the class of set systems closed under intersection and union. Finally, we will call distributive regular set system any distributive lattice given under the form $\mathcal{O}(P)$, where $P$ is endowed with the appropriate partial order relation $\leqslant$.

We present now the following inclusion diagram where these set systems structures fit into each other (see Fig. 1).

\section{Proposition 2}

(1) The class of Jordan-Dedekind set systems strictly includes regular set systems.

(2) The class of regular set systems strictly includes regular set lattices.

(3) The class of regular set lattices strictly includes convex geometries and antimatroids.

(4) The intersection of the classes of convex geometries and antimatroids coincides with the class of distributive regular set systems. 
(5) The class of distributive regular set systems strictly includes distributive regular set systems isomorphic to direct products of linear lattices.

(6) The class of direct products linear lattices strictly includes Boolean lattices.

Proof: We show the successive inclusions (1) to (4), (5) and (6) being well known or evident.

(1) is clear since for any regular set system, for any two coalitions $S$ and $T$ such that $S \subseteq T$, all maximal chains from $S$ to $T$ have clearly $t-s+1$ elements. However, the reverse property is not true. Indeed, it is self-evident that for any $n \geq 3$, the poset $\{\emptyset, 1,2, \ldots, n, N\}$ is a JordanDedekind set system but is not regular, since its maximal chains have length 2 and should have length $n$.

About (2), we have only to show that the inclusion is strict: for $n=4$, we easily see that the set system $\mathcal{N}:=\{\emptyset, 1,2,13,23,14,24,123,124,1234\}$ is regular but $\{1\}$ and $\{2\}$ have no supremum thus $(N, \mathcal{N})$ is not a lattice.

By a simple induction using $(C 1)$, we show that any maximal chain of a convex geometry $(N, \mathcal{N})$ has necessarily length $n$. By Proposition $1,(N, \mathcal{N})$ is a regular set system. This holds for an antimatroid, by the duality principle. Besides, the convex geometry and the antimatroid are lattices $(\mathcal{N}, \subseteq, \vee, \cap, N, \emptyset)$ and $(\mathcal{N}, \subseteq, \cup, \wedge, N, \emptyset)$ where $A \vee B:=\cap\{C \in \mathcal{N} \mid A \cup B \subseteq C\}$ and $A \wedge B:=\cup\{C \in \mathcal{N} \mid A \cap B \subseteq C\}$, respectively. Conversely, the set system $\{\emptyset, 1,2,13,23,123\}$ is a regular lattice but is neither a convex geometry nor an antimatroid. Thus, (3) is shown.

Now, let $(N, \leqslant)$ be any poset and $L:=\mathcal{O}(N)$ be the distributive lattice of all downsets of $(N, \leqslant)$. As said above, it is known that the union and the intersection of any two downsets is also a downset. Furthermore, it is clear that condition (C1) holds since from any downset $S \neq N$ of $(N, \leqslant)$, adding a minimal element of the restricted poset $(N \backslash S, \leqslant)$ in $S$ leaves $S$ a downset. By withdrawing a maximal element of $S \neq \emptyset$, the dual condition (A1) holds. Conversely, if a set system is a convex geometry and an antimatroid, then supremum and infimum laws are union and intersection, which immediately implies the distributivity law. Thus (4) is shown.

Remark that (5) and (6) are seen as set system inclusions in the sense that for any direct product of linear lattices $L$, there is a regular set system $(N, \mathcal{N})$ that is isomorphic to $L$. In addition, for any Boolean lattice $\mathcal{B}$, there is an integer $n$ such that $\mathcal{B}$ is isomorphic to $2^{N}$.

We call regular game any game defined on a regular set system, that is to say, any mapping $v$ defined over a regular set system $(N, \mathcal{N})$ such that $v(\emptyset)=0$. We denote by $\mathcal{G}(\mathcal{N})$ the $\mathbb{R}$-vector space of games over the set system $(N, \mathcal{N})$. Remark that for the Boolean lattice $\mathcal{N}:=2^{N}, \mathcal{G}(\mathcal{N})$ is the set of classical cooperative games.

Considering a regular set system $(N, \mathcal{N})$, the following mappings

$$
\begin{aligned}
\delta_{S}: \mathcal{N} & \rightarrow \mathbb{R} \\
A & \mapsto\left\{\begin{array}{l}
1 \text { if } A=S, \\
0 \text { otherwise }
\end{array}\right.
\end{aligned}
$$

form a special collection of games in $\mathcal{G}(\mathcal{N})$, that are called identity games, for $S \in \mathcal{N}$. Note that the mapping $\delta_{\emptyset}$ is not a game since $\delta_{\emptyset}(\emptyset)=1$.

We also introduce symmetric games, whose worths depend only on the cardinality of the coalitions, and equidistributed games of $\mathcal{G}(\mathcal{N})$, which are regular games $v$ that are both symmetric and additive, that is, worths $v(S)$ are proportional to $s$ :

$$
\exists \nu \in \mathbb{R} \text { such that } \forall S \in \mathcal{N}, v(S)=\nu \cdot s .
$$




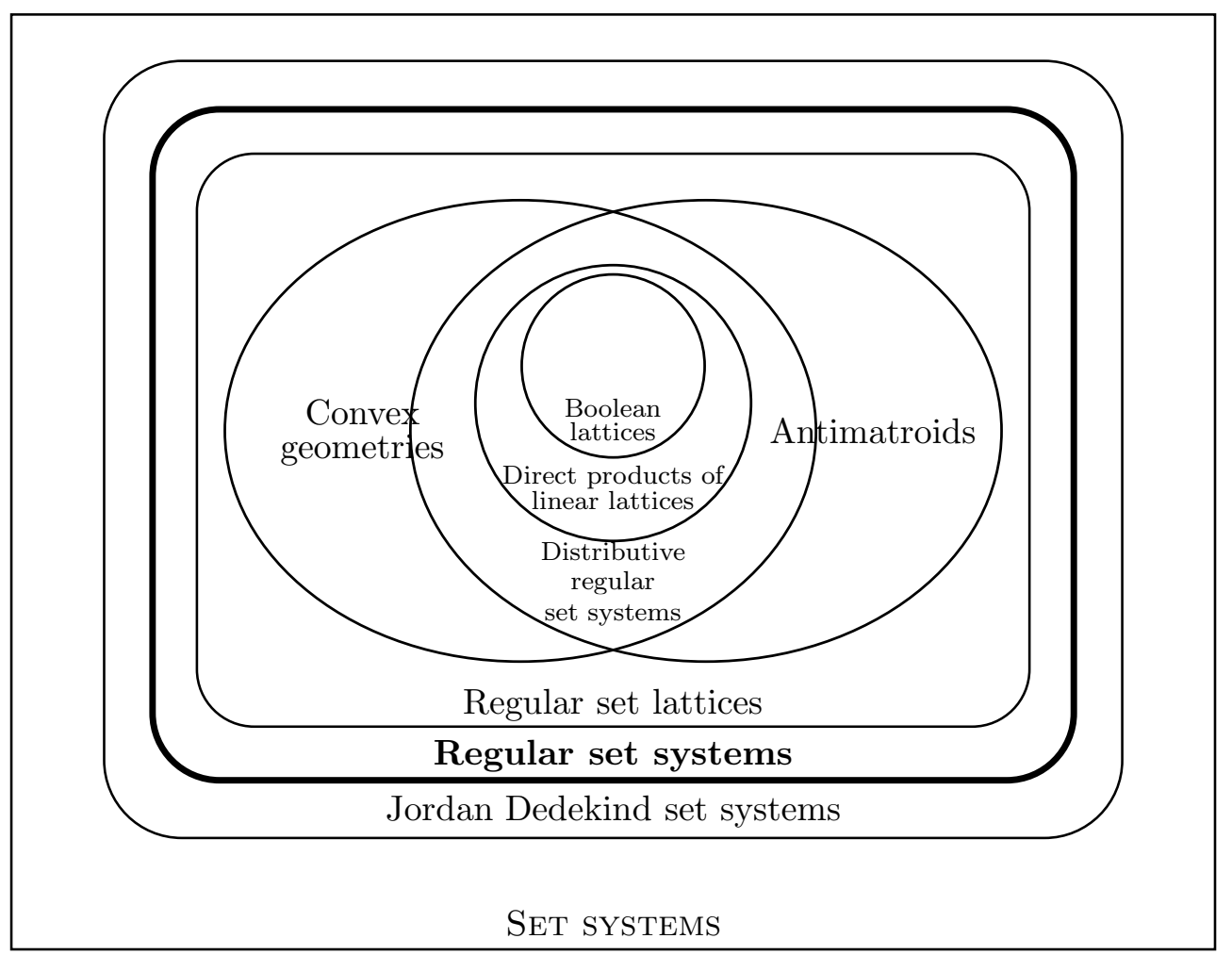

Figure 1: Inclusion diagram of set systems

As a consequence of Proposition 2, the material we propose in what follows, is convenient as well for games on convex geometries (cf. Bilbao (2000), chap.7), and thus for games with precedence constraints (Faigle and Kern (1992)), where feasible coalitions of players are the only ones that respect a given precedence structure on the set of players: let $(N, \leqslant)$ be a partially ordered set of players, where $\leqslant$ is a relation of precedence in the sense that $i \leqslant j$ if the presence of $j$ enforces the presence of $i$ in any coalition $S \subseteq N$. Hence, a coalition of $N$ is a subset $S$ of $N$ such that $i \in S$ and $j \leqslant i$ entails $j \in S$. Consequently, the collection $\mathcal{C}(N)$ of all coalitions of $N$ is the collection of all downsets of $(N, \leqslant)$, which is a distributive regular set system.

\section{Probabilistic and efficient values}

From now on, $(N, \mathcal{N})$ refers to a regular set system. A value on $\mathcal{G}(\mathcal{N})$ is a mapping $\Phi: \mathcal{G}(\mathcal{N}) \rightarrow$ $\mathbb{R}^{n}$ that associates to each game $v$ a vector $\left(\Phi^{1}(v), \ldots, \Phi^{n}(v)\right)$, where the real number $\Phi^{i}(v)$ represents the payoff to player $i$ in the game $v$. The Shapley value for cooperative games $\Phi_{S h}$ is well known (Shapley (1953)).

$$
\forall v \in \mathcal{G}\left(2^{N}\right), \forall i \in N, \quad \Phi_{S h}^{i}(v):=\sum_{S \subseteq N \backslash i} \frac{s !(n-s-1) !}{n !}(v(S \cup i)-v(S)) .
$$

Following the work of Weber (1988), Bilbao has defined and axiomatized a class of values for games defined over convex geometries, the probabilistic values. It is possible to define such values for regular games.

First, we denote by $S+i$ the coalition $S \cup i$ whenever $S \not \supset i$. Thus, writing $S+i \in \mathcal{N}$ infers two relations: $i \notin S$ and $S \cup i \in \mathcal{N}$. Similarly, $S-i$ denotes the coalition $S \backslash i$ and infers $S \ni i$. 
Definition $2 A$ value $\Phi$ on $\mathcal{G}(\mathcal{N})$ is a probabilistic value if there exists for each player $i$, a collection of real numbers $\left\{p_{S}^{i} \mid S \in \mathcal{N}, S+i \in \mathcal{N}\right\}$ satisfying $p_{S}^{i} \geq 0$ and $\sum_{S \in \mathcal{N} \mid S+i \in \mathcal{N}} p_{S}^{i}=1$ such that

$$
\Phi^{i}(v)=\sum_{S \in \mathcal{N} \mid S+i \in \mathcal{N}} p_{S}^{i}(v(S \cup i)-v(S))
$$

for every game $v \in \mathcal{G}(\mathcal{N})$.

If no condition is required for real numbers $p_{S}^{i}$, then we call $\Phi$ a marginalist value.

Observe that for a probabilistic value, the participation of player $i$ is assessed to be a weighted average of his marginal contribution $v(S \cup i)-v(S)$ whenever $i$ joins coalition $S$ (provided that $S \cup i$ is a feasible coalition), $p_{S}^{i}$ being the subjective probability that $i$ joins $S$.

In a cooperative game, it is assumed that all players decide to cooperate among them and form the grand coalition $N$. This leads to the problem of distributing the amount $v(N)$ among them. In this case, a value $\Phi$ is efficient if it satisfies:

Efficiency axiom (E): $\forall v \in \mathcal{G}(\mathcal{N}), \quad \sum_{i=1}^{n} \Phi^{i}(v)=v(N)$.

We consider also the following axioms.

Linearity axiom (L): $\forall i \in N, \forall v, w \in \mathcal{G}(\mathcal{N}), \forall \alpha \in \mathbb{R}, \quad \Phi^{i}(\alpha v+w)=\alpha \Phi^{i}(v)+\Phi^{i}(w)$.

Player $i$ is a null player when his contribution to all coalitions $S \cup i \in \mathcal{N}$ formed with his incorporation to $S \in \mathcal{N}$ has no effect.

Definition 3 A player $i \in N$ is null for $v \in \mathcal{G}(\mathcal{N})$ if

$\forall S \in \mathcal{N}$ such that $S+i \in \mathcal{N}, \quad v(S \cup i)=v(S)$.

Player $i$ is dummy for $v \in \mathcal{G}(\mathcal{N})$ if

$\forall S \in \mathcal{N}$ such that $S+i \in \mathcal{N}, \quad v(S \cup i)-v(S)= \begin{cases}v(i), & \text { if } i \in \mathcal{N} \\ 0, & \text { else. }\end{cases}$

Null axiom (N): If player $i$ is null for $v$, then $\Phi^{i}(v)=0$.

The dummy axiom of Bilbao, introduced to axiomatize games on convex geometries, is:

Dummy axiom (D): If player $i$ is dummy for $v$, then $\Phi^{i}(v)=v(i)$, whenever $i \in \mathcal{N}$ and 0 otherwise.

Monotonicity axiom (M): If the game $v \in \mathcal{G}(\mathcal{N})$ is monotonic, that is to say,

$S \subseteq T$ implies $v(S) \leq v(T)$ for all $S, T \in \mathcal{N}$, then the values $\Phi^{i}$ are nonnegative.

Let us present the axiomatization of probabilistic values for games on regular set systems, as already seen in Bilbao (2000).

Proposition 3 Let $\Phi$ a value on $\mathcal{G}(\mathcal{N})$. Under axioms $(\boldsymbol{L})$ and $(\boldsymbol{N})$, $\Phi$ is a marginalist value.

Proof: First, under (L), for all $i \in N$, there is a unique collection of real numbers $\left\{c_{S}^{i} \mid S \in\right.$ $\mathcal{N}, S \neq \emptyset\}$ such that

$$
\Phi^{i}(v)=\sum_{S \in \mathcal{N}, S \neq \emptyset} c_{S}^{i} v(S)
$$


for every game $v \in \mathcal{G}(\mathcal{N})$. Indeed, the collection of identity games is clearly a basis of $\mathcal{G}(\mathcal{N})$ since every game $v$ can be written as

$$
v=\sum_{S \in \mathcal{N}, S \neq \emptyset} v(S) \delta_{S},
$$

in a unique way. By axiom $(\mathbf{L})$,

$$
\Phi^{i}(v)=\sum_{S \in \mathcal{N}, S \neq \emptyset} v(S) \Phi^{i}\left(\delta_{S}\right) .
$$

Now, this formula can also write

$$
\Phi^{i}(v)=\sum_{\substack{S \in \mathcal{N} \mid \\ S-i \in \mathcal{N} \text { or } S+i \in \mathcal{N}}} v(S) \Phi^{i}\left(\delta_{S}\right)+\sum_{\substack{S \in \mathcal{N} \mid \\ S-i \notin \mathcal{N} \text { and } S+i \notin \mathcal{N}}} v(S) \Phi^{i}\left(\delta_{S}\right) .
$$

Next, assume that $S \in \mathcal{N}, i \notin S$ and $S+i \notin \mathcal{N}$ (resp. $S \in \mathcal{N}, i \in S$ and $S-i \notin \mathcal{N}$ ). Thus $i$ is null for $\delta_{S}$ and, by $(\mathbf{N}), \Phi^{i}\left(\delta_{S}\right)=0$. Then, the second part of the above sum vanishes. Therefore

$$
\begin{aligned}
\Phi^{i}(v) & =\sum_{S \in \mathcal{N} \mid S+i \in \mathcal{N}}\left[v(S) \Phi^{i}\left(\delta_{S}\right)+v(S \cup i) \Phi^{i}\left(\delta_{S \cup i}\right)\right] \\
& =\sum_{S \in \mathcal{N} \mid S+i \in \mathcal{N}}[v(S \cup i)-v(S)] \Phi^{i}\left(\delta_{S \cup i}\right)+\sum_{S \in \mathcal{N} \mid S+i \in \mathcal{N}} v(S)\left[\Phi^{i}\left(\delta_{S}\right)+\Phi^{i}\left(\delta_{S \cup i}\right)\right] \\
& =\sum_{S \in \mathcal{N} \mid S+i \in \mathcal{N}} \Phi^{i}\left(\delta_{S \cup i}\right)[v(S \cup i)-v(S)]+\sum_{S \in \mathcal{N} \mid S+i \in \mathcal{N}} v(S) \Phi^{i}\left(\delta_{S}+\delta_{S \cup i}\right) .
\end{aligned}
$$

Since $i$ is null for $\delta_{S}+\delta_{S \cup i}$ whenever $S \in \mathcal{N}$ and $S+i \in \mathcal{N}$, we conclude that $\Phi^{i}\left(\delta_{S}+\delta_{S \cup i}\right)=0$ by $(\mathbf{N})$. Thus

$$
\Phi^{i}(v)=\sum_{S \in \mathcal{N} \mid S+i \in \mathcal{N}} \Phi^{i}\left(\delta_{S \cup i}\right)[v(S \cup i)-v(S)] .
$$

Observe now that the dummy axiom implies the null axiom since a null player $i$ is a particular dummy player satisfying $v(i)=0$. Bilbao (2000) has shown that values for games over convex geometries (that are particular regular games) which satisfy axioms (L), (D), (M) and (E), are precisely the efficient probabilistic values.

We improve now this result by weakening the set of axioms and considering more general structures.

Theorem 4 Let $\Phi$ be a value on $\mathcal{G}(\mathcal{N})$. Under axioms $(\boldsymbol{L}),(\boldsymbol{N}),(\boldsymbol{M})$ and $(\boldsymbol{E}), \Phi$ is a probabilistic and an efficient value.

Proof: Whenever $i \notin S$, we denote by $p_{S}^{i}$ the coefficient $\Phi^{i}\left(\delta_{S \cup i}\right)$ of formula (3) above.

Let choose some $T \in \mathcal{N}$ and define the game of $\mathcal{G}(\mathcal{N})$

$$
\hat{u}_{T}(S)= \begin{cases}1, & \text { if } S \supsetneq T \\ 0, & \text { else. }\end{cases}
$$


By definition, $\hat{u}_{T}$ is monotonic. Letting $i \in N$ and $T \in \mathcal{N}$ such that $T+i \in \mathcal{N}$, under (L), (N) and $(\mathbf{M})$, we get:

$$
\begin{aligned}
\Phi^{i}\left(\hat{u}_{T}\right) & =\sum_{S \in \mathcal{N} \mid S+i \in \mathcal{N}} p_{S}^{i}\left(\hat{u}_{T}(S \cup i)-\hat{u}_{T}(S)\right) \\
& =p_{T}^{i} \geq 0 .
\end{aligned}
$$

Lastly, it remains to show under the efficiency axiom that for all $i \in N$, the collections of number $\left\{p_{S}^{i} \mid S \in \mathcal{N}: S+i \in \mathcal{N}\right\}$ form probability distributions, so that we could conclude to the result. For any $i \in N$, let us consider the game

$$
u_{i}(S)= \begin{cases}1, & \text { if } S \ni i \\ 0, & \text { else }\end{cases}
$$

Then, on the one hand, we have under $(\mathbf{E})$,

$$
\sum_{j=1}^{n} \Phi^{j}\left(u_{i}\right)=u_{i}(N)=1 .
$$

And on the other hand,

$$
\begin{aligned}
\sum_{j=1}^{n} \Phi^{j}\left(u_{i}\right) & =\sum_{j=1}^{n} \sum_{S \in \mathcal{N} \mid S+j \in \mathcal{N}} p_{S}^{j}\left(u_{i}(S \cup j)-u_{i}(S)\right) \\
& =\sum_{S \in \mathcal{N} \mid S+i \in \mathcal{N}} p_{S}^{i}\left(u_{i}(S \cup i)-u_{i}(S)\right)+\sum_{j \in N \backslash i} \sum_{S \in \mathcal{N} \mid S+j \in \mathcal{N}} p_{S}^{j}\left(u_{i}(S \cup j)-u_{i}(S)\right) \\
& =\sum_{S \in \mathcal{N} \mid S+i \in \mathcal{N}} p_{S}^{i},
\end{aligned}
$$

since differences of the first sum always worth 1 whereas differences of the second one vanish. This finishes the proof.

We present now an important result about marginalist values, already known for convex geometries (Bilbao (2000), chap.7).

Proposition 5 Let $\Phi$ be a marginalist value on $\mathcal{G}(\mathcal{N})$, defined by

$$
\Phi^{i}(v)=\sum_{S \in \mathcal{N} \mid S+i \in \mathcal{N}} p_{S}^{i}(v(S \cup i)-v(S))
$$

for every game $v$ and for all $i \in N$, where $p_{S}^{i}$ are real numbers. Then $\Phi$ satisfies the efficiency axiom if and only if

$$
\begin{aligned}
\sum_{i \in N \mid i \in \mathcal{N}} p_{\emptyset}^{i} & =\sum_{i \in N \mid N \backslash i \in \mathcal{N}} p_{N \backslash i}^{i}=1, \\
\sum_{i \in N \mid S-i \in \mathcal{N}} p_{S \backslash i}^{i} & =\sum_{i \in N \mid S+i \in \mathcal{N}} p_{S}^{i},
\end{aligned}
$$

for all $S \in \mathcal{N} \backslash\{\emptyset, N\}$. 
Proof: For every $v \in \mathcal{G}(\mathcal{N})$, we compute the sum of the values $\Phi^{i}(v)$.

$$
\begin{aligned}
\sum_{i=1}^{n} \Phi^{i}(v) & =\sum_{i=1}^{n} \sum_{S \in \mathcal{N} \mid S+i \in \mathcal{N}} p_{S}^{i}(v(S \cup i)-v(S)) \\
& =\sum_{S \in \mathcal{N}}\left(\sum_{i \in N \mid S-i \in \mathcal{N}} p_{S \backslash i}^{i}-\sum_{i \in N \mid S+i \in \mathcal{N}} p_{S}^{i}\right) v(S) \\
& =\sum_{\substack{S \in \mathcal{N} \mid \\
S \neq \emptyset, N}}\left(\sum_{i \in N \mid S-i \in \mathcal{N}} p_{S \backslash i}^{i}-\sum_{i \in N \mid S+i \in \mathcal{N}} p_{S}^{i}\right) v(S)+\left(\sum_{i \in N \mid N-i \in \mathcal{N}} p_{N \backslash i}^{i}\right) v(N) .
\end{aligned}
$$

If the coefficients satisfy (4) and (5), then it is clear that $\Phi$ satisfies the efficiency axiom.

Conversely, fix $T \in \mathcal{N}$ such that $T \neq \emptyset, N$ and consider the identity game $\delta_{T}$. The efficiency axiom straightforwardly implies that $\sum_{i=1}^{n} \Phi^{i}\left(\delta_{T}\right)=0$. Applying the above equality to $\delta_{T}$, we have

$$
\sum_{i=1}^{n} \Phi^{i}\left(\delta_{T}\right)=0=\sum_{i \in N \mid T-i \in \mathcal{N}} p_{T \backslash i}^{i}-\sum_{i \in N \mid T+i \in \mathcal{N}} p_{T}^{i},
$$

that is to say (5) is proven. If $T=N$, then the equality becomes

$$
\sum_{i=1}^{n} \Phi^{i}\left(\delta_{N}\right)=1=\sum_{i \in N \mid N-i \in \mathcal{N}} p_{N \backslash i}^{i}
$$

which partially proves (4). Finally, consider the game $\hat{u}_{\emptyset}:=\sum_{T \in \mathcal{N}, T \neq \emptyset} \delta_{T}$. Then

$$
\begin{aligned}
\sum_{i=1}^{n} \Phi^{i}\left(\hat{u}_{\emptyset}\right)=1 & =\sum_{i=1}^{n} \sum_{S \in \mathcal{N} \mid S+i \in \mathcal{N}} p_{S}^{i}\left(\hat{u}_{\emptyset}(S \cup i)-\hat{u}_{\emptyset}(S)\right) \\
& =\sum_{i \in N \mid i \in \mathcal{N}} p_{\emptyset}^{i} \hat{u}_{\emptyset}(i)=\sum_{i \in N \mid i \in \mathcal{N}} p_{\emptyset}^{i}
\end{aligned}
$$

which achieves the proof.

\section{The Shapley value for regular games}

If we focus now on the particular case of classical cooperative games, we know that Weber has characterized the Shapley value on $\mathcal{G}\left(2^{N}\right)$ as the unique probabilistic value satisfying the well known symmetry axiom, assuming that the coefficients of the value should not depend on the labelling of the elements of $N$, that is a very natural property.

The fundamental idea of the symmetry axiom rests on permutations of players. Symmetry could be naturally defined in regular games. Unfortunately, this generalization has a very limited interest: apart from particular cases of regular set systems, players generally cannot be permuted, which leaves this axiom ineffective.

Faigle and Kern (1992) attempted to generalize the Shapley value for their games under precedence constraints, which are games over distributive regular set systems (cf. Proposition 2), and thus particular regular games: in their framework, maximal chains correspond to what they call feasible ranking of players. For that, the hierarchical strength axiom is introduced, which is actually difficult to interpret. 
We propose a different approach for the axiomatization of the Shapley value on $\mathcal{G}(\mathcal{N})$. Consider first the classical Shapley value on $\mathcal{G}\left(2^{N}\right)$ where $p_{S}^{i}:=\frac{s !(n-s-1) !}{n !}($ cf. (1)).

Observing that for any subsets $A, A+i+j \subseteq N$, the equality $p_{A}^{i}+p_{A \cup i}^{j}=p_{A}^{j}+p_{A \cup j}^{i}$ holds. One may wonder if this property is sufficient to form the Shapley value from a probabilistic and efficient value. Actually, the answer is positive. Furthermore, one can generalize it to any case of game defined on a regular set system.

Indeed, let us consider again a 3-players game whose communication graph links player 1 with player 2 , and player 2 with player 3 , but not 1 with 3 . We remind that the set of feasible coalitions is thus $\{\emptyset, 1,2,3,12,23, N\}$. Besides, let $v$ be any equidistributed game (Section 2). Since $v$ is additive as well as symmetric, the sum of the marginal contributions of players for forming any feasible coalition should not depend on the chosen order. Considering for instance the maximal chains $(\emptyset, 1,12)$ and $(\emptyset, 2,12)$ then the following should hold:

$$
p_{\emptyset}^{1} v(1)+p_{1}^{2}(v(12)-v(1))=p_{\emptyset}^{2} v(2)+p_{2}^{1}(v(12)-v(2)),
$$

that is equivalent to

$$
p_{\emptyset}^{1}+p_{1}^{2}=p_{\emptyset}^{2}+p_{2}^{1},
$$

since $v$ is equidistributed. A similar result is also obtained by considering the formation of coalition 23, according to whether player 2 is the first to join or not the coalition. Likewise, from coalition $\{2\}$, there are two ways to achieve the grand coalition, which is written:

$$
p_{2}^{1}(v(12)-v(2))+p_{12}^{3}(v(123)-v(12))=p_{2}^{3}(v(23)-v(2))+p_{23}^{1}(v(123)-v(23)),
$$

that is equivalent to

$$
p_{2}^{1}+p_{12}^{3}=p_{2}^{3}+p_{23}^{1} .
$$

In this way, assuming that the value satisfies also the efficiency axiom, by combining the above equations with those given by (4) and (5), we have to solve a linear system, then we obtain a unique solution for the vector of coefficients $\left(p_{\emptyset}^{1}, p_{\emptyset}^{2}, p_{1}^{2}, p_{1}^{3}, p_{2}^{1}, p_{2}^{3}, p_{12}^{3}, p_{13}^{2}, p_{23}^{1}\right)$ :

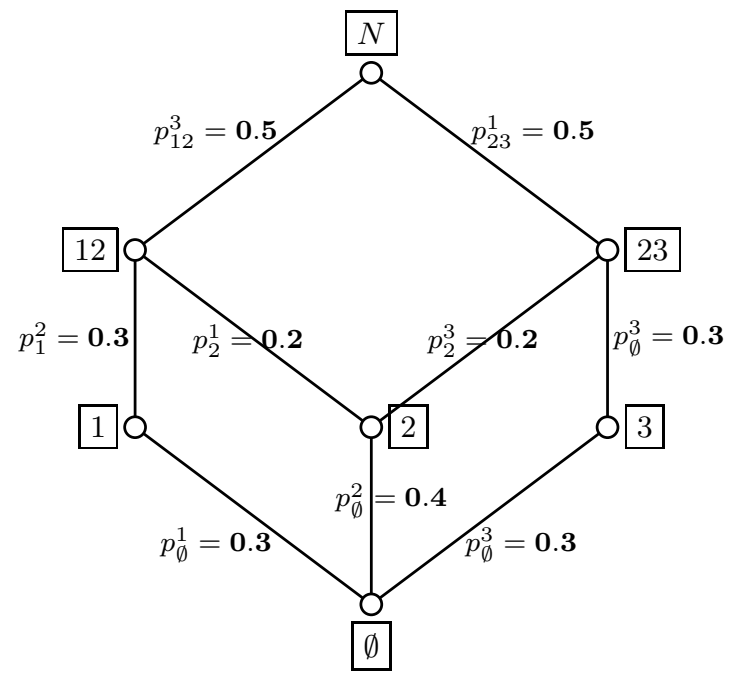

So as to formalize this, we introduce the following material. Let $A, B$ be any two coalitions of $\mathcal{N}$ such that $A \subseteq B$, and $\mathcal{C}$ be a maximal chain from $A$ to $B$. Let $\sigma$ be any permutation 
of $N$ such that $\mathcal{C}$ may be written $\left(A, S_{\sigma(a+1)}, \ldots, S_{\sigma(b)}\right)$, where $S_{i}$ denotes the first coalition containing player $i$ in the chain. Note that $\sigma$ is unique if $A=\emptyset$ and $B=N$. Besides, for any marginalist value $\Phi$ whose coefficients are given by (2) and any game $v \in \mathcal{G}(\mathcal{N})$, we denote the cumulative sum of marginal contributions of players of $B \backslash A$ along $\mathcal{C}$ by:

$$
m_{\Phi}^{\mathcal{C}}(v):=\sum_{i \in B \backslash A} p_{S_{i} \backslash i}^{i}\left(v\left(S_{i}\right)-v\left(S_{i} \backslash i\right)\right) .
$$

Thus, for such a game, the cumulative sum of expected marginal contributions of involved players of $B \backslash A$ should not depend on the considered maximal chain from $A$ to $B$, since the path taken from $A$ to $B$ has no effect on the successive increasing worth $v(C), A \subseteq C \subseteq B$.

In this spirit, we propose the following axiom.

Regularity axiom $(\mathbf{R})$ : For any equidistributed game $v \in \mathcal{G}(\mathcal{N})$, for any couple of maximal chains $\mathcal{C}_{1}, \mathcal{C}_{2}$ of $(N, \mathcal{N})$, then $m_{\Phi}^{\mathcal{C}_{1}}(v)=m_{\Phi}^{\mathcal{C}_{2}}(v)$.

We call regular value any marginalist value satisfying the regularity axiom.

Actually, the regularity axiom may be seen as a generalization of the Shapley's symmetry axiom. The next result confirms this view, and gives a generalization of the Shapley's result asserting the unicity of the value $\Phi$ under the linearity axiom, the null axiom, the efficiency axiom and the regularity axiom.

Theorem 6 Let $(N, \mathcal{N})$ be a regular set system. Then there is a unique efficient regular value $\Phi_{K}$ on $\mathcal{G}(\mathcal{N})$.

To show this result, we need to introduce new material and definitions, that is done in the next section and in Appendix A.

\section{The Shapley value in the framework of network theory}

Considering results of previous section, let us fix a regular set system $(N, \mathcal{N})$ and a marginalist value $\Phi$ on $\mathcal{G}(\mathcal{N})$, and let $\left\{p_{S}^{i} \mid i \in N, S \in \mathcal{N}\right.$ s.t. $\left.S+i \in \mathcal{N}\right\}$ be the set of associated coefficients. We associate to any regular set system $(N, \mathcal{N})$ an electrical network, that is, an interconnection of electrical components such as resistors. Precisely, we consider the mapping

$$
(N, \mathcal{N}) \mapsto \mathcal{E}(N, \mathcal{N})
$$

where $\mathcal{E}(N, \mathcal{N})$ is built in this way: nodes of $\mathcal{E}(N, \mathcal{N})$ are simply the elements of $\mathcal{N}$, whereas its branches are directed wires given by the couples $b_{S}^{i}:=(S, S+i)$ of $(N, \mathcal{N})$. Note that at this stage, $\mathcal{E}(N, \mathcal{N})$ may be seen as the Hasse diagram of $(N, \mathcal{N})$, since the $b_{S}^{i}$ 's are given by the covering relation $\prec$ of $(N, \mathcal{N})$. We complete the building by adding another branch $b_{N, \emptyset}$ by connecting the node $N$ with the node $\emptyset$.

In $\mathcal{E}(N, \mathcal{N})$, we call circuit $^{1}$, any sequence $\left(b_{1}, \ldots, b_{m}\right)$ of branches such that for all $k=$ $2, \ldots, m-1, b_{k}$ shares a common node with $b_{k-1}$ and its other node with $b_{k+1}$, and such that $b_{1}$ and $b_{m}$ are also connected. For convenience, we also may write a circuit as a sequence of $m$ nodes $\left(S_{0}, S_{1}, \ldots, S_{m}=S_{0}\right)$.

Now, one can attribute to the branches $b_{S}^{i}\left(\right.$ resp. $\left.b_{N, \emptyset}\right)$ some weights $I_{S \rightarrow S \cup i}$ and $V_{S \rightarrow S \cup i}$ (resp. $I_{N \rightarrow \emptyset}$ and $V_{N \rightarrow \emptyset}$ ), where $I_{b}$ 's are the worths of a directed commodity flowing in the branches called electrical current, and where $V_{b}$ 's are worths proportional to currents $I_{b}$ 's: $V_{b}$ 's

\footnotetext{
${ }^{1}$ This terminology shall also be used for $(N, \mathcal{N})$
} 
are called potential drops and satisfy the well-known Ohm's law: $V_{b}=R_{b} \cdot I_{b}$, where $R_{b}$ is said to be the resistance of the non-oriented branch $b$. In our framework, we assign for any electrical network a unitary resistance to every branch $b_{S}^{i}$, so that $V_{b}=I_{b}$. Precisely, the necessary and sufficient following conditions for the electrical current and potential drops must be satisfied:

First Kirchhoff's law: The sum of all currents entering a node is equal to the sum of all currents leaving the node.

Second Kirchhoff's law: The directed sum of the electrical potential drops around a circuit must be zero.

Therefore, let us now assign to any branch $b_{S}^{i}$ the coefficient $p_{S}^{i}$ of the marginalist value $\Phi$, with $I_{N \rightarrow \emptyset}:=1$. Thus Proposition 5 asserts that $\Phi$ satisfies the efficiency axiom if and only if the first Kirchhoff's law is satisfied in $\mathcal{E}(N, \mathcal{N})$.

We also establish a straight link between the second Kirchhoff's law and the regularity axiom in Corollary 8. In this respect, for any circuit $\mathcal{M}:=\left(b_{1}, \ldots, b_{m}\right)$ on $(N, \mathcal{N})$, for $j=1, \ldots, m$, if $b_{j}$ represents the couple $(S, S+i)$, we denote by $\bar{p}_{j}=\bar{p}_{S}^{i}$ the signed coefficient of $\Phi$ associated to $b_{j}$ relatively to its orientation in $\mathcal{M}$ :

$$
\bar{p}_{S}^{i}:=\left\{\begin{aligned}
p_{S}^{i} & \text { if in } \mathcal{M}, b_{j} \text { is directed in accordance with } \subseteq, \\
-p_{S}^{i} & \text { otherwise. }
\end{aligned}\right.
$$

As a consequence, the second Kirchhoff's law fitted for the marginalist value $\Phi$ may be expressed by

Circuit property: For any circuit $\left(b_{1}, \ldots, b_{m}\right)$ of $(N, \mathcal{N}), \sum_{j=1}^{m} \bar{p}_{j}=0$.

We call potential over $(N, \mathcal{N})$ any real-valued mapping $\mathbf{V}$ defined on $\mathcal{N}$ satisfying for any coefficient $p_{S}^{i}$

$$
p_{S}^{i}=\mathbf{V}(S+i)-\mathbf{V}(S) .
$$

Proposition 7 Let $\Phi$ be a marginalist value on $\mathcal{G}(\mathcal{N})$ and $\left\{p_{S}^{i}|i \in N, S \in \mathcal{N}| S+i \in \mathcal{N}\right\}$ be the set of associated coefficients. Then the circuit property holds if and only if there exists a potential over $(N, \mathcal{N})$.

Moreover, for a given instance of coefficients $p:=\left(p_{S}^{i}\right)_{(S, S+i) \in \mathcal{N}^{2}}$ satisfying the circuit property, there is a unique potential $\mathbf{V}$ vanishing at $\emptyset$. We call it the potential associated to $p$ and grounded on $\emptyset$, and denote it by $\mathbf{V}_{\mathbf{0}}^{p}$.

Proof: The sufficiency condition is clear. Indeed, let $\left(b_{1}, \ldots, b_{m}\right)$ be a circuit of $(N, \mathcal{N})$, where the $b_{j}$ 's belong to $E$ and $\left(S_{0}, S_{1}, \ldots, S_{m}=S_{0}\right)$ be the same circuit expressed in terms of coalitions. Then

$$
\begin{aligned}
\sum_{j=1}^{m} V_{b_{j}} & =\sum_{j=1}^{m}\left(\mathbf{V}\left(S_{j}\right)-\mathbf{V}\left(S_{j-1}\right)\right) \\
& =\mathbf{V}\left(S_{m}\right)-\mathbf{V}\left(S_{0}\right) \\
& =0
\end{aligned}
$$

Conversely, assume that the circuit property holds. Let us define by induction on the cardinality of the coalitions, the following correspondance:

$$
\begin{gathered}
\mathbf{V}(\emptyset):=0, \\
\forall S \in \mathcal{N} \backslash\{\emptyset\}, \text { and } i \in S, \mathbf{V}(S):=\mathbf{V}(S \backslash i)+p_{S \backslash i}^{i} .
\end{gathered}
$$


Then by definition, $\mathbf{V}$ has the required property of a potential. Thus it remains to show that this mapping is properly defined. Indeed, let $S \in \mathcal{N}$ and $i, k$ two distinct players in $S$. By an inductive argument, we make the assumption that $\mathbf{V}$ is properly defined on $\{T \in \mathcal{N} \mid t<s\}$. Thus we must show that $\mathbf{V}(S \backslash i)+p_{S \backslash i}^{i}=\mathbf{V}(S \backslash k)+p_{S \backslash k}^{k}$. Let $S_{0}$ be any maximal element of the set $\{T \in \mathcal{N} \mid T \subseteq S \backslash i, T \subseteq S \backslash k\}$ and $m:=s-s_{0}(m \geq 2)$. Thus there is a circuit $\mathcal{M}=\left(b_{1}, \ldots, b_{2 m}\right)$ such that $b_{1}$ is directed from $S_{0}, b_{m}=b_{S \backslash i}^{i}$ and $b_{m+1}=b_{S \backslash k}^{k}$. By induction, we easily compute $\mathbf{V}(S \backslash i)=\mathbf{V}\left(S_{0}\right)+\sum_{j=1}^{m-1} p\left(b_{j}\right)$ and $\mathbf{V}(S \backslash k)=\mathbf{V}\left(S_{0}\right)+\sum_{j=m+2}^{2 m} p\left(b_{j}\right)$. Besides, if we denote by $\left(S_{0}, S_{1}, \ldots, S_{2 m}=S_{0}\right)$ the same circuit in terms of coalitions, we get by the circuit property

$$
\begin{aligned}
\sum_{j=1}^{2 m} V_{b_{j}} & =0 \\
\text { iff } \sum_{j=1}^{m} p\left(b_{j}\right) & =\sum_{j=m+1}^{2 m} p\left(b_{j}\right) \\
\text { iff } \mathbf{V}\left(S_{0}\right)+\sum_{j=1}^{m-1} p\left(b_{j}\right)+p_{S \backslash i}^{i} & =\mathbf{V}\left(S_{0}\right)+\sum_{j=m+2}^{2 m} p\left(b_{j}\right)+p_{S \backslash k}^{k} \\
\text { iff } \mathbf{V}(S \backslash i)+p_{S \backslash i}^{i} & =\mathbf{V}(S \backslash k)+p_{S \backslash k}^{k} .
\end{aligned}
$$

Now, we show that if there are two mappings $\mathbf{V}_{1}$ and $\mathbf{V}_{2}$ satisfying (6), then they are the same up to an additive constant. Let us denote by $c$ the real number $\mathbf{V}_{2}(\emptyset)-\mathbf{V}_{1}(\emptyset)$. For any $S \in \mathcal{N}$, there is a maximal chain $\left(T_{0}=\emptyset, T_{1}, \ldots, T_{s}=S\right)$ from $\emptyset$ to $S$. Thus, by denoting $i_{j}$ the singleton $T_{j} \backslash T_{j-1}$, we get by (6)

$$
\mathbf{V}_{k}(S)=\mathbf{V}_{k}\left(T_{s}\right)=\mathbf{V}_{k}\left(T_{s-1}\right)+p_{T_{s-1}}^{i_{s}}=\mathbf{V}_{k}\left(T_{s-2}\right)+p_{T_{s-2}}^{i_{s-1}}+p_{T_{s-1}}^{i_{s}}=\cdots=\mathbf{V}_{k}(\emptyset)+\sum_{j=1}^{s} p_{T_{j-1}}^{i_{j}}
$$

for $k=1,2$. Thus, $\mathbf{V}_{2}(S)-\mathbf{V}_{1}(S)=\mathbf{V}_{2}(\emptyset)-\mathbf{V}_{1}(\emptyset)=c$. As a consequence, by fixing $\mathbf{V}$ on any vertex, we have a unique possibility for the potential, which gives $\mathbf{V}_{\mathbf{0}}^{p}$ if $\emptyset$ is assigned to 0 .

Lastly, we deduce the following result, asserting that $\Phi$ satisfies the regularity axiom if and only if the second Kirchhoff's law is satisfied in $\mathcal{E}(N, \mathcal{N})$.

Corollary 8 Let $\Phi$ be a marginalist value on $\mathcal{G}(\mathcal{N})$ :

$$
\Phi^{i}(v)=\sum_{S \in \mathcal{N} \mid S+i \in \mathcal{N}} p_{S}^{i}(v(S \cup i)-v(S))
$$

where $p_{S}^{i}$ are real numbers, for all $i \in N$. Then $\Phi$ satisfies the regularity axiom if and only if the circuit property holds.

Proof: The regularity axiom means that along any maximal chain of $(N, \mathcal{N})$, for an equidistributed game $v$, the cumulative sum of marginal contributions of players do not depend on the considered maximal chain. Since $v$ is equidistributed, worths $v(S)$ only depend on a multiplicative constant, thus it is equivalent to only consider coefficients along the chain.

Assuming firstly that the circuit property holds, we consider any potential $\mathbf{V}$ associated to the coefficients $p_{S}^{i}$ 's. Therefore, if $\mathcal{C}:=\left(\emptyset, S_{\sigma(1)}, \ldots, S_{\sigma(n)}=N\right)$ is any maximal chain of $(N, \mathcal{N})$ 
and $v$ is any equidistributed game defined by $v(S):=\nu \cdot s, \forall S \in \mathcal{N}$, we obtain

$$
\begin{aligned}
m_{\Phi}^{\mathcal{C}}(v) & =\nu \sum_{j=1}^{n}\left(\mathbf{V}\left(S_{\sigma(j)}\right)-\mathbf{V}\left(S_{\sigma(j)} \backslash \sigma(j)\right)\right) \\
& =\nu \cdot(\mathbf{V}(N)-\mathbf{V}(\emptyset))
\end{aligned}
$$

which does not depend on $\mathcal{C}$, and thus the regularity axiom holds.

Conversely, if the regularity axiom holds, we build the same correspondance than in the proof of Proposition 7, that is to say

$$
\begin{gathered}
\mathbf{V}(\emptyset):=0 \\
\forall S \in \mathcal{N} \backslash\{\emptyset\}, \text { and } i \in S, \mathbf{V}(S):=\mathbf{V}(S \backslash i)+p_{S \backslash i}^{i}
\end{gathered}
$$

Then by a similar argument than in the proof of Proposition 7, we show that $\mathbf{V}$ is a potential, which will implies that the circuit property holds. Once again, we just have to show that $\mathbf{V}$ is properly defined, that is to say, by making again the assumption that $\mathbf{V}$ is properly defined on $\{T \in \mathcal{N}|t<| S \mid\}$, where $S \in \mathcal{N}$ and $i, k \in S(i \neq k)$, we must show that $\mathbf{V}(S \backslash i)+p_{S \backslash i}^{i}=\mathbf{V}(S \backslash k)+p_{S \backslash k}^{k}$. Let $\mathcal{C}^{1}:=\left(b_{1}^{1}, \ldots, b_{n}^{1}\right)$ and $\mathcal{C}^{2}:=\left(b_{1}^{2}, \ldots, b_{n}^{2}\right)$ be any two maximal chains such that $b_{s}^{1}=(S \backslash i, S), b_{s}^{2}=(S \backslash k, S)$ and $b_{j}^{1}=b_{j}^{2}$ for every $j>s$. We easily verify that $\mathbf{V}(S \backslash i)=\sum_{j=1}^{s-1} p\left(b_{j}^{1}\right)$ and $\mathbf{V}(S \backslash k)=\sum_{j=1}^{s-1} p\left(b_{j}^{2}\right)$. Besides, by the regularity axiom applied on $\mathcal{C}^{1}$ and $\mathcal{C}^{2}$

$$
\begin{gathered}
\sum_{j=1}^{n} p\left(b_{j}^{1}\right)=\sum_{j=1}^{n} p\left(b_{j}^{2}\right) \\
\text { iff } \mathbf{V}(S \backslash i)+p_{S \backslash i}^{i}+\sum_{j=s+1}^{n} p\left(b_{j}^{1}\right)=\mathbf{V}(S \backslash k)+p_{S \backslash k}^{k}+\sum_{j=s+1}^{n} p\left(b_{j}^{2}\right) \\
\operatorname{iff} \mathbf{V}(S \backslash i)+p_{S \backslash i}^{i}=\mathbf{V}(S \backslash k)+p_{S \backslash k}^{k} .
\end{gathered}
$$

To sum up, we could say with a slight abuse of language that the unique efficient regular value on $\mathcal{G}(\mathcal{N})$, is also the unique marginalist value satisfying the Kirchhoff's laws in the sense that it satisfies (4), (5) and the circuit property. That is why we may call $\Phi_{K}$ the Kirchhoff's value or also the Shapley-Kirchhoff value.

This being introduced, we have now a sufficient material to show Theorem 6 , that is made in Appendix A.

\section{Shapley-Kirchhoff value and monotonicity axioms}

At this point of the work, a natural question arises about another property of the ShapleyKirchhoff value. Indeed, $\Phi_{K}$ satisfies the linearity axiom, the null axiom, the efficiency axiom and the regularity axiom. However, we have no information about the monotonicity axiom, that would make of $\Phi_{K}$ a probabilistic value. With this in mind, we present the following handy short result. 
Lemma 9 Let $\Phi$ be a marginalist value on $\mathcal{G}(\mathcal{N})$, defined by

$$
\Phi^{i}(v)=\sum_{S \in \mathcal{N} \mid S+i \in \mathcal{N}} p_{S}^{i}(v(S \cup i)-v(S))
$$

for every game $v$ and for all $i \in N$, where $p_{S}^{i}$ are real numbers. Then $\Phi$ satisfies the monotonicity axiom if and only if all $p_{S}^{i}$ 's are nonnegative.

Proof: Let $v$ be a game on $\mathcal{G}(\mathcal{N})$. If all $p_{S}^{i}$ 's are nonnegative and $v$ monotonic, $\Phi^{i}(v)$ is clearly nonnegative for any $i \in N$.

Conversely, let $i \in N$ and $S \in \mathcal{N}$ with $S+i \in \mathcal{N}$, such that $p_{S}^{i}<0$. Let $\hat{u}_{S} \in \mathcal{G}(\mathcal{N})$ be the unanimity game defined by

$$
\forall T \in \mathcal{N}, \quad \hat{u}_{S}(T)=\left\{\begin{array}{l}
1 \text { if } T \supsetneq S \\
0 \text { otherwise. }
\end{array}\right.
$$

Thus, $u_{S}$ is clearly monotonic, nevertheless $\Phi^{i}(v)=p_{S}^{i}<0$.

In the light of this result, for $\Phi_{K}$ to satisfy the monotonicity axiom, we should show that coefficients $p_{S}^{i}$ 's are nonnegative. Actually, it turns out that there are regular set systems for which the Shapley-Kirchhoff value does not satisfy the monotonicity axiom, as the counterexample given in Appendix B proves it.

Nevertheless, it appears that there are other kinds of monotonicity axioms in the framework of cooperative games, as Young (1985) worked out. Indeed, monotonicity is a general principle of fair division which states that as the underlying data of a problem change, the solution should change in a parallel fashion. We give the following monotonicity axioms adapted to our framework (cf. p.7).

A frequently encountered form of monotonicity is aggregate monotonicity. This principle states that if the worth of the coalition of the whole increases, while the worth of all other coalitions remains fixed, then no player should get less than before.

Aggregate monotonicity axiom $(\mathbf{A M})$ : Let $v, w$ two games in $\mathcal{G}(\mathcal{N})$ such that $w(N) \geq v(N)$ and $w(S)=v(S)$ for other coalitions $S \in \mathcal{N}$. Then, $\forall i \in N, \quad \Phi^{i}(w) \geq$ $\Phi^{i}(v)$.

Coalitional monotonicity is satisfied if an increase in the worth of a particular coalition implies no decrease in the allocation to any member of that coalition. Thus the following axiom is stronger than the previous one.

Coalitional monotonicity axiom (CM): Let $S \in \mathcal{N}$ and $v, w$ two games in $\mathcal{G}(\mathcal{N})$ such that $w(S) \geq v(S)$ and $w(T)=v(T)$ for other coalitions $T \in \mathcal{N}$. Then, $\forall i \in S, \quad \Phi^{i}(w) \geq \Phi^{i}(v)$.

Coalitional monotonicity refers to monotonic changes in the absolute worth of the coalitions a given player. There are also situations where the worth of coalitions containing a given player $i$ increase relatively to the worth of coalitions not containing $i$ :

Strong monotonicity axiom (SM): Let $v, w$ two games in $\mathcal{G}(\mathcal{N})$ and a player $i$ such that for every $S \in \mathcal{N}$ satisfying $S+i \in \mathcal{N}, w(S \cup i)-w(S) \geq v(S \cup i)-v(S)$. Then $\Phi^{i}(w) \geq \Phi^{i}(v)$. 
Having described these monotonicity axioms, we give results in context of games over regular set systems.

Proposition 10 Under linearity and null axioms, (M), (SM) and (CM) are equivalent. In addition, these axioms are strictly stronger than (AM), whatever the regular set system is.

Proof: We successively show that $(\mathbf{M}) \Leftrightarrow(\mathbf{S M})$ and $(\mathbf{M}) \Leftrightarrow(\mathbf{C M})$.

- Due to Lemma 9, if (M) holds, then (SM) also holds since $\Phi^{i}(v)$ depends only on the marginal contributions of player $i$. Conversely, let $i \in N$ and $S \in \mathcal{N}$ with $S+i \in$ $\mathcal{N}$, such that $p_{S}^{i}<0$, so that $(\mathbf{M})$ is not true. Let $v, w$ be any two games such that $w(S \cup i)-w(S)=v(S \cup i)-v(S)+1$, and for any other coalition $T \in \mathcal{N}$ satisfying $T+i \in \mathcal{N}, w(T \cup i)-w(T)=v(T \cup i)-v(T)$. Then assumption of (SM) is satisfied but $\Phi^{i}(w)-\Phi^{i}(v)=p_{S}^{i}<0$, that is to say (SM) does not hold.

- Due to Lemma 9, if (M) holds then (CM) clearly holds. Indeed, if only the worth of a coalition $S$ increases in the game $v$, then for any player $i$ of the coalition, in $\Phi^{i}(v)$, the associated marginal contribution $p_{S \backslash i}^{i}(v(S)-v(S \backslash i))$ increases, and other marginal contribution remain the same. Conversely, let us assume that (M) is not satisfied, that is to say there is a player $i \in N$ and a coalition $S \in \mathcal{N}$ with $S+i \in \mathcal{N}$, such that $p_{S}^{i}<0$. Let $v, w$ be any two games such that $w(S \cup i)=v(S \cup i)+1$, and $w(T)=v(T)$ for any other coalition $T \in \mathcal{N}$. Then $\Phi^{i}(w)-\Phi^{i}(v)=p_{S}^{i}<0$, that is to say (SM) does not hold.

Second part of the result is clear and is an immediate consequence of Lemma 11.

Thus, as the aggregate monotonicity axiom is weaker than the classical one, one may wonder if the Shapley-Kirchhoff values satisfies it. The answer is actually positive and rests on the following characterization.

Lemma 11 Let $\Phi$ be a marginalist value on $\mathcal{G}(\mathcal{N})$, defined by

$$
\Phi^{i}(v)=\sum_{S \in \mathcal{N} \mid S+i \in \mathcal{N}} p_{S}^{i}(v(S \cup i)-v(S))
$$

for every game $v$ and for all $i \in N$, where $p_{S}^{i}$ are real numbers. Then $\Phi$ satisfies the aggregate monotonicity axiom if and only if for every $i$ in $N$ such that $N \backslash i \in \mathcal{N}, p_{N \backslash i}^{i}$ is nonnegative.

Proof: The sufficient condition derives from Lemma 9. Indeed, if all $p_{N \backslash i}^{i}$ 's are nonnegative and $v, w$ any two games having the same worths, except for $N$ with $w(N) \geq v(N)$, then $\Phi^{j}(w)-\Phi^{j}(v)=p_{N \backslash j}^{j}(w(N)-v(N))$ or vanishes, depending on whether $N \backslash j$ is a coalition of $\mathcal{N}$ or not. Conversely, let us assume that there is a player $i \in N$ satisfying $N \backslash i \in \mathcal{N}$, such that $p_{N \backslash i}^{i}<0$. Let $v, w$ be any two games such that $w(N)=v(N)+1$ and $w(T)=v(T)$ for any other coalition. Then $\Phi^{i}(w)-\Phi^{i}(v)=p_{N \backslash i}^{i}<0$. Thus the necessary condition is satisfied.

Finally, we show this final result, whose proof uses the Maximum Principle applied to a valued graph (see Appendix A).

Theorem 12 For any regular set system $(N, \mathcal{N})$, the Shapley-Kirchhoff value $\Phi_{K}$ satisfies the aggregate monotonicity axiom. 


\section{APPENDICES}

\section{A Dirichlet problem on a graph and potentials}

The problem of finding the currents on the branches of a resistor network, an entering current being given, is easy to solve if seen as the solution of a Dirichlet problem associated to that network (Colin de Verdière (1998)). Thus, we introduce now a few rudimentary notions of discrete potential theory, in order to prove Theorems 6 and 12 .

Let $G=(V, E)$ be a non-oriented connected graph where the set of vertices $V$ is randomly divided into two distinct categories, a non-empty set of boundary points $V_{0}$ and the set of interior points $V_{1}=V \backslash V_{0}$. For any interior point $x$, we denote by $d(x)$ the degree of vertex $x$ in $G$, that is to say $d(x):=\mid\{y \in V \mid\{x, y\} \in E)\} \mid$. A function $f$ defined on $V$ is said to be harmonic on $G$ if, for points $x$ in $V_{1}$, it has the averaging property

$$
f(x)=\frac{\sum_{\{x, y\} \in E} f(y)}{d(x)},
$$

with no restriction on the values of $f$ at the boundary points.

Now the problem of finding a harmonic function given its boundary values is called the Dirichlet problem, and the Uniqueness Principle for the Dirichlet problem asserts that there cannot be two different harmonic functions having the same boundary values. We approach the Uniqueness Principle by way of the Maximum Principle for harmonic functions.

Maximum Principle. A harmonic function $f$ defined on $V$ takes on its maximum value $M$ and its minimum value $m$ on the boundary.

Proof: If $M$ is the maximum value of $f$ and if $f(x)=M$ for $x$ an interior point, then since $f(x)$ is the average of the values of $f$ at its neighbors, these values must all equal $M$ also. By working our way, repeating this argument at every step, we eventually reach a boundary point $x_{0}$ for which we can conclude that $f\left(x_{0}\right)=M$. That same argument works for the minimum value $m$.

Uniqueness Principle. If $f$ and $g$ are harmonic on $V$ such that $f=g$ on $V_{0}$, then $f(x)=g(x)$ for all $x \in V$.

Proof: Let $h:=f-g$. Then if $x$ is any interior point,

$$
\frac{\sum_{\{x, y\} \in E} h(y)}{d(x)}=\frac{\sum_{\{x, y\} \in E} f(y)}{d(x)}-\frac{\sum_{\{x, y\} \in E} g(y)}{d(x)}=f(x)-g(x)=h(x) .
$$

Therefore $h$ is a harmonic function which vanishes on $V_{0}$, and hence, by the Maximum Principle, the maximum and minimum values of $h$ are 0 . Thus $h$ is identically null, and $f=g$.

Once again, let us consider graph $G=\left(\left(V_{0}, V_{1}\right), E\right)$ as an electrical network, where $V=$ $V_{0} \cup V_{1}$ is the set of nodes, with $\left|V_{0}\right|=2$, and $E$ is the set of branches, each of them having a unit resistance. If a voltage $\mathbf{V}$ is given on $V_{0}$, and satisfies the first Kirchhoff's law for interior points:

$$
\forall x \in V_{1}, \sum_{\{x, y\} \in E}(\mathbf{V}(y)-\mathbf{V}(x))=0
$$


then $\mathbf{V}$ is precisely a harmonic function. Indeed, since all resistances of the branches are unitary, the current in the oriented branch $(x, y)$ is expressed by the potential drop $\mathbf{V}(y)-\mathbf{V}(x)$.

Let $x$ be an interior node, then solving (8) for $\mathbf{V}(x)$ straightforwardly gives the averaging property (7), and by the Uniqueness Principle, we conclude that $f=\mathbf{V}$. It remains to show that such a function exists, which rests on basic linear algebra. Indeed, by expressing (8) (or (7)) for each interior point, we easily obtain a linear system $\Gamma\left(\mathbf{V}_{\mathbf{1}}\right)=B, \Gamma$ being a $v_{1} \times v_{1}$ morphism, $\mathbf{V}_{\mathbf{1}}$ being the vector of unknown variables $(\mathbf{V}(x))_{x \in V_{1}}$, and where the right-hand member $B$ depends only on the values taken by $\mathbf{V}$ on the boundary points. $\Gamma$ being an injective endomorphism, then it is also bijective. Thus by

$$
\mathbf{V}_{\mathbf{1}}=\Gamma^{-1}(B)
$$

the existence of $\mathbf{V}$ follows.

\section{Proof: [Theorem 6]}

Let $V_{0}:=\{\emptyset, N\}$ and $V_{1}:=\mathcal{N} \backslash V_{0}$. By the Uniqueness Principle, given a real number $R$, there is a unique potential $\mathbf{V}$ defined on $\mathcal{N}$, such that $\mathbf{V}(\emptyset)=0, \mathbf{V}(N)=R$, and satisfying the first Kirchhoff's law on $V_{1}$. Moreover, we know by (9) that $\mathbf{V}$ is linearly dependent on $R$, that is to say, proportional to $R$ :

$$
\forall S \in \mathcal{N}, \mathbf{V}(S)=\mathbf{V}_{\mathbf{0}}^{\mathbf{1}}(S) \cdot R
$$

where $\mathbf{V}_{\mathbf{0}}^{\mathbf{1}}$ is the required potential for $R=1$. Thus one can adjust the value of $R$ so that

$$
\sum_{S \succ \emptyset} \mathbf{V}(S)=1
$$

Indeed, let $C_{\mathcal{N}}:=\sum_{S \succ \emptyset} \mathbf{V}_{\mathbf{0}}^{\mathbf{1}}(S)$ and $R_{\mathcal{N}}:=C_{\mathcal{N}}^{-1}$ (note that $C_{\mathcal{N}}$ is non null since by the argument used for Maximum Principle, all $\mathbf{V}_{\mathbf{0}}^{\mathbf{1}}(S)$ 's, $S \neq \emptyset$, are necessarily strictly positive). Thus for $R=R_{\mathcal{N}}$, (10) holds. We denote by $\mathbf{V}_{\mathbf{0}}$ the associated potential, that is to say, satisfying $\mathbf{V}_{\mathbf{0}}(\emptyset)=0$ and $\mathbf{V}_{\mathbf{0}}(N)=R_{\mathcal{N}}$.

Now, for any $(S, S+i) \in \mathcal{N}$, define $p_{S}^{i}:=\mathbf{V}_{\mathbf{0}}(S+i)-\mathbf{V}_{\mathbf{0}}(S)$. Therefore, the marginalist value associated to the coefficients $p_{S}^{i}$ 's is an efficient regular value. Indeed, by Proposition 7 , the circuit property holds, and thus by Corollary 8, the regularity axiom also holds. Besides, by Proposition 5, the efficency axiom holds, (5) being equivalent to (8), and (4) being expressed by (10) on the one hand, and by conservation of the flow pattern $\left(p_{S}^{i}\right)_{(S, S+i) \in \mathcal{N}^{2}}$ on the other hand.

Lastly, coefficients $p_{S}^{i}$ 's being determined by the unique potential grounded on $\emptyset$ and satisfying (10), the unicity of $\Phi_{K}$ is also shown.

\section{Proof: [Theorem 12]}

Let $\mathbf{V}$ be a potential associated to the coefficients $p_{S}^{i}$ 's of $\Phi_{K}$. The source node of the electrical network being $\emptyset, \mathbf{V}$ is necessarily greater on bound $N$ than bound $\emptyset$. Then by the Maximum Principle, $\forall i \in N$ such that $N \backslash i \in \mathcal{N}, p_{N \backslash i}^{i}=\mathbf{V}(N)-\mathbf{V}(N \backslash i)$ is nonnegative. By Lemma 11, the result follows. 


\section{B Example of regular set system for which $\Phi_{K}$ does not satisfy the monotonicity axiom}

Let $N:=\left\{\alpha, \beta, \gamma, 1,2, \ldots, n^{\prime}\right\}$ be the set of players, with $N^{\prime}:=\left\{1, \ldots, n^{\prime}\right\}$ and $n^{\prime} \geq 1$. We make the assumption that the set of coalitions $\mathcal{N}$ is defined by

$$
\mathcal{N}:=\{\emptyset, N, N \backslash \beta, N \backslash \gamma\} \cup \bigcup_{i=0}^{n^{\prime}}\{\{\alpha, 1,2, \ldots, i\}\} \cup \bigcup_{S \in 2^{N^{\prime}}}\{S \cup \beta\} .
$$

Then $(N, \mathcal{N})$ is a regular set system. The associated Shapley-Kirchhoff value defined over $\mathcal{G}(\mathcal{N})$ satisfies the monotonicity axiom if and only if $n^{\prime}<5$. Indeed, we show that if it is not the case, coefficient $p_{N^{\prime} \cup \alpha}^{\beta}$ is negative, which corresponds to a negative current in the associated directed branch $\left(N^{\prime} \cup \alpha, N^{\prime} \cup \alpha \beta\right)$ (cf. Section 5). Fig. 2 is given with $n^{\prime}=5$, where the bold line represents the above mentionned branch.

Proof: The first point is to verify that $(N, \mathcal{N})$ is a regular set system. By checking that each coalition $S$ of $\mathcal{N}$ has all its successors (for inclusion order) $T$ satisfying $|T \backslash S|=1$, we have the result.

We compute now the different coefficients $p_{S}^{i}$ of the Shapley-Kirchhoff $\Phi_{K}$ value associated to the regular set system $(N, \mathcal{N})$. Let us denote by $x$ the coefficient $p_{\emptyset}^{\beta}$ and $y$ the coefficient $p_{N^{\prime} \cup \alpha}^{\beta}$. On the one hand, we consider the sub-order $\mathcal{N}_{\beta}^{\prime}$ induced by all vertices associated to coalitions including $\beta$ and included in $N^{\prime} \cup \beta . \mathcal{N}_{\beta}^{\prime}$ being isomorphic to the Boolean lattice $2^{N^{\prime}}$, any permutation of $N^{\prime}$ leaves unchanged $\mathcal{N}_{\beta}^{\prime}$, and since the circuit property is a symmetric rule (in the sense that labels of players have no importance for it), the coefficients computed for the edges of $\mathcal{N}_{\beta}^{\prime}$ are proportional to the coefficients of the classical Shapley value over $\mathcal{G}(\mathcal{N})$ whenever $\mathcal{N}=2^{N^{\prime}}$. Indeed, for any subset $S$ of $N^{\prime}$ and any $i \in N^{\prime} \backslash S$, let us denote by $x_{s}^{1}$ the coefficient $p_{S \cup \beta}^{i}$. By Proposition 5, we get

$$
\begin{aligned}
& x_{0}^{1}=\frac{x}{n^{\prime}}, \\
& s \cdot x_{s-1}^{1}=\left(n^{\prime}-s\right) \cdot x_{s}^{1}, \quad \forall s \in\left\{1, \ldots, n^{\prime}-1\right\},
\end{aligned}
$$

which immediately conducts by induction on $s$ to

$$
x_{s}^{1}=\frac{s !\left(n^{\prime}-s-1\right) !}{n^{\prime} !} \cdot x, \quad \forall s \in\left\{0, \ldots, n^{\prime}-1\right\} .
$$

Remark that the left coefficient also known under the form $p_{s}^{1}\left(n^{\prime}\right)$, is well-known since being a coefficient of Shapley for games with $n^{\prime}$ players (cf. (1)).

Naturally, we also get $p_{N^{\prime} \cup \beta}^{\alpha}=\sum_{i=1}^{n^{\prime}} x_{n^{\prime}-1}^{1}=x$.

On the other hand, also by Proposition 5, we successively deduce:

- On node $\emptyset, p_{\emptyset}^{\alpha}=1-x$.

- On nodes $\alpha 1 \ldots i, i \in N^{\prime}, p_{\alpha}^{1}=p_{\alpha 1}^{2}=\cdots=p_{\alpha 12 \ldots\left(n^{\prime}-1\right)}^{n^{\prime}}=1-x$.

- On nodes $N^{\prime} \cup \alpha, N \backslash \beta, N \backslash \gamma, p_{N^{\prime} \cup \alpha}^{\gamma}=p_{N \backslash \beta}^{\beta}=1-x-y$, and $p_{N \backslash \gamma}^{\gamma}=x+y$.

Now, let $\mathcal{M}_{1}$ be the circuit $\left(\emptyset, \alpha, \alpha 1, \alpha 12, \ldots, N^{\prime} \cup \alpha, N \backslash \gamma, N^{\prime} \cup \beta, \ldots, \beta 12, \beta 1, \beta, \emptyset\right)$ and $\mathcal{M}_{2}$ be the circuit $\left(N, N \backslash \gamma, N^{\prime} \cup \alpha, N \backslash \beta, N\right)$. Then by the circuit property applied to these two circuits, we have the system:

$$
\left\{\begin{array}{ll}
\left(n^{\prime}+1\right)(1-x)+y-x-\sum_{s=0}^{n^{\prime}-1} x_{s}^{1}-x & =0 \\
-(x+y)-y+2(1-x-y) & =0
\end{array} .\right.
$$


By denoting $\varsigma\left(n^{\prime}\right)$ the sum of the coefficients of Shapley $\sum_{s=0}^{n^{\prime}-1} p_{s}^{1}\left(n^{\prime}\right)$, the above system writes

$$
\left\{\begin{array}{ll}
\left(n^{\prime}+3+\varsigma\left(n^{\prime}\right)\right) x-y & =n^{\prime}+1 \\
3 x+4 y & =2
\end{array},\right.
$$

and has for determinant the strictly positive number $\Delta:=4\left(n^{\prime}+\varsigma\left(n^{\prime}\right)\right)+15$. Thus, we obtain

$$
\begin{aligned}
& x=\frac{4 n^{\prime}+6}{\Delta} \text { and } \\
& y=\frac{2 \varsigma\left(n^{\prime}\right)-n^{\prime}+3}{\Delta} .
\end{aligned}
$$

For all the above $p_{S}^{i}$ 's given in terms of $x$ and $y$, except for $p_{N^{\prime} \cup \alpha}^{\beta}=y$, we straightforwardly verify that $p_{S}^{i}>0$, for all $n^{\prime} \geq 1$. Thus it remains to find condition on $n^{\prime}$ so that $y$ is nonnegative. For $n^{\prime} \leq 4$, it can be checked that $2 \varsigma\left(n^{\prime}\right)-n^{\prime}+3$ is positive. Besides, $\forall n^{\prime} \geq 1, \forall s \in$ $\left\{0, \ldots, n^{\prime}-1\right\}, p_{s}^{1}\left(n^{\prime}\right)=\frac{s !\left(n^{\prime}-s-1\right) !}{n^{\prime} !} \leq \frac{\left(s+\left(n^{\prime}-s-1\right)\right) !}{n^{\prime} !}=\frac{1}{n^{\prime}}$, the inequality being strict whenever $s \neq 0, n^{\prime}-1$. Thus, $\forall n^{\prime}>2, \varsigma\left(n^{\prime}\right)=\sum_{s=0}^{n^{\prime}-1} p_{s}^{1}\left(n^{\prime}\right)<1$. Moreover,

$$
\Delta \cdot y / 2=\varsigma\left(n^{\prime}\right)-\left(n^{\prime}-3\right) / 2,
$$

where $\left(n^{\prime}-3\right) / 2 \geq 1, \forall n^{\prime} \geq 5$. As a consequence, $\varsigma\left(n^{\prime}\right)-\left(n^{\prime}-3\right) / 2<0, \forall n^{\prime} \geq 5$, and so is $y$. The result finally follows, by Lemma 9 .

In particular, whenever $n^{\prime}=5$, which corresponds to the regular system of Fig. $2, \Phi_{K}$ is not monotonic, with $p_{\alpha 12345}^{\beta}=-\frac{14}{557}$.

\section{Acknowledgment}

The first author is very grateful to Professor H. Narayanan for his kind and helpful attitude, giving much of his time in the investigation of the counterexample of Appendix B.

\section{References}

Bilbao, J.M., (2000). Cooperative Games on Combinatorial Structures, Kluwer Academic Publishers.

Bilbao, J.M., Edelman, P., (2000). The Shapley value on convex geometries. Discrete Applied Mathematics, 103, 33-40.

Birkhoff, G., (1967). Lattice theory (3d ed.), American Mathematical Society.

Calvo, E., Lasage, J., van den Nouweland, A., (1999). Values of games with probabilistic graphs. Mathematical Social Sciences, 37, 79-95.

Choquet, G., (1953). Theory of capacities. Annales de l'Institut Fourier, 5, 131-295.

Colin de Verdière, Y., (1998). Spectres de graphes, Collection S.M.F.

Deo, N., (1974). Graph theory with applications to engineering and computer science, PrenticeHall.

Doyle, P.G., Snell, J.L., (1984). Random walks and electric networks, The mathematical association of America.

Dubey, P., Neyman, A., Weber, R.J., (1981). Value theory without efficiency. Mathematics of Operations Research, 6(1), 122-128. 


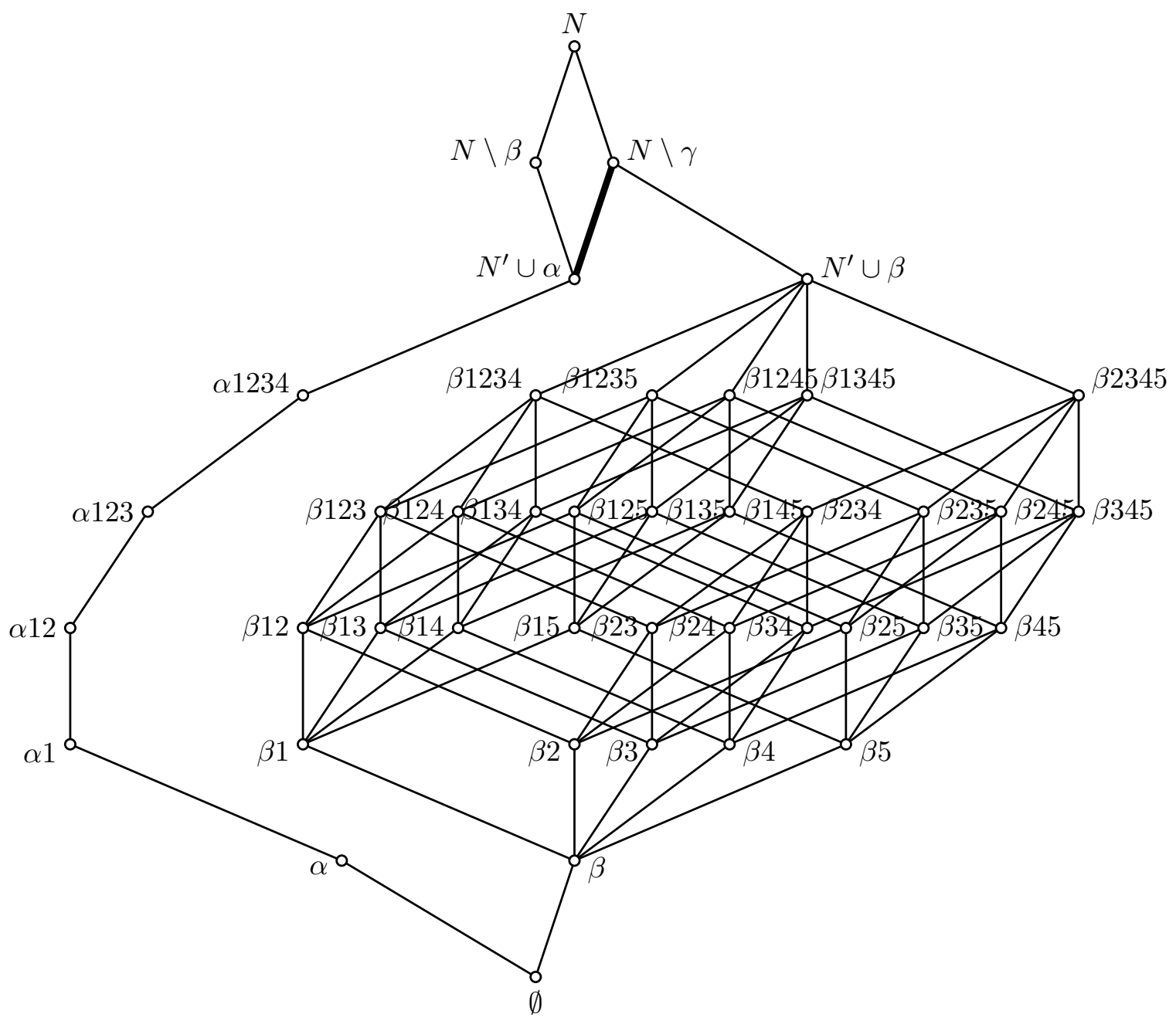

Figure 2: A regular set system where (M) does not hold for $\Phi_{K}$

Faigle, U., (1989). Cores of games with restricted cooperation. ZOR-Methods and Models of Operations Research, 33(6), 405-422.

Faigle, U., Kern, W., (1992). The Shapley value for cooperative games under precedence constraints. International Journal of Game Theory, 21, 249-266.

Grabisch, M., Lange, F., (2007). Games on lattices, multichoice games and the Shapley value: a new approach. Mathematical Methods of Operations Research, 65, 153-167.

Grabisch, M., Marichal, J.L., Roubens, M., (2000). Equivalent representations of set functions. Mathematics of Operations Research, 25(2), 157-178.

Honda, A., Grabisch, M., (2006). Entropy of capacities on lattices and set systems. Information Sciences, $176(23), 3472-3489$.

Hwang, Y.A., Liao, Y.H., (2008). Potential approach and characterizations of a Shapley value in multi-choice games. Mathematical Social Sciences, 56, 321-335.

Labreuche, Ch., (2002). Interaction indices for games with forbidden coalitions. In: 9th int. conf. on Information Processing and Management of Uncertainty in Knowledge-Based Systems (IPMU'2002), p. 529-534, Annecy, France.

Myerson, R.B., (1977). Graphs and cooperation in games. Mathematics of Operation Research, 2(3), 225-229. 
Shapley, L.S., (1953). A value for n-person games. In: Kuhn, H.W., Tucker, A.W. (Eds.), Contributions to the Theory of Games, Vol. II, p. 307-317. Princeton University Press.

van den Brink, R., van den Laan, G., Pruzhansky, V., (2004). Harsanyi power solutions for graph-restricted games. Tinbergen Institute Discussion Paper, (TI 04-095/1).

Weber, R.J., (1988). Probabilistic values for games. In: Roth, A.E. (Ed.), The Shapley Value. Essays in Honor of Lloyd S. Shapley, p. 101-119. Cambridge University Press.

Young, H.P., (1985). Monotonic solutions of cooperative games. International Journal of Game Theory, 14, 65-72. 